\title{
Severe cranial deformity following cerebrospinal fluid diversion in an adolescent with osteogenesis imperfecta
}

\author{
Winson S. Ho, MD, ${ }^{1,2}$ and John A. Jane Jr., MD² \\ ${ }^{1}$ Surgical Neurology Branch, National Institute of Neurological Disorders and Stroke, National Institutes of Health, Bethesda, \\ Maryland; and 'Department of Neurosurgery, University of Virginia, Charlottesville, Virginia
}

\begin{abstract}
Osteogenesis imperfecta $(\mathrm{OI})$ is an inherited connective tissue disorder that causes bone fragility and deformity. Neurological manifestations, including macrocephaly and hydrocephalus, have been reported. Increased vascular fragility or bleeding diathesis also predisposes OI patients to intracranial hemorrhage. The development of chronic subdural fluid collections or hydrocephalus may require CSF diversion. The authors report a previously unrecognized complication of CSF diversion in a patient with $\mathrm{OI}$, that is, a delayed severe cranial deformity, presumably due to over-shunting. In addition to the cosmetic concern, the deformity caused severe headaches and tenderness. The patient underwent craniectomy and titanium mesh cranioplasty, which resulted in the complete resolution of symptoms. This report raises the possibility that over-shunting in patients with $\mathrm{Ol}$ could predispose to the formation of cranial deformity requiring surgical intervention.
\end{abstract}

https://thejns.org/doi/abs/10.3171/2018.4.PEDS18109

KEYWORDS osteogenesis imperfecta; shunt; cranial deformity; craniofacial

$\mathrm{O}$ STEOGENESIS imperfecta $(\mathrm{OI})$ is a rare connective disorder ( 1 case in 15,000-20,000 births) resulting in skeletal fragility and growth deficiency. ${ }^{3}$ Most patients present early in life with multiple bone fractures that are often misdiagnosed as nonaccidental trauma. Neurological manifestations of OI have been reported, including ventriculomegaly with or without hydrocephalus ${ }^{1,11}$ and basilar invagination causing myelopathy and/or brainstem compression. ${ }^{7,11}$ In addition, researchers have associated OI with vascular fragility or bleeding diathesis, which thus predisposes OI patients to intracranial hemorrhage. ., $, 8-10,12$ Cerebrospinal fluid diversion in patients with OI is indicated in the setting of recurrent subdural hematoma or hydrocephalus. We present the case of an adolescent who, after shunting, developed a severe cranial deformity in a large area of Wormian bone formation at her lambdoid sutures, which was treated with craniectomy and cranioplasty.

\section{Case Report}

History and Examination

This 17-year-old female with a history of OI had been adopted from Russia as a child. She first presented to the University of Virginia emergency room when she was 7 years old with complaints of headache following a minor head trauma several weeks earlier. Head CT demonstrated a chronic left-sided subdural hematoma and an incidental right middle cranial fossa cyst (Fig. 1A and B), which was managed conservatively and resolved on follow-up (Fig. 1C). At that time, it was noted that she had mild ventriculomegaly, which was thought to represent cerebral atrophy due to a history of traumatic brain injury or malnutrition in infancy. Clinically, she fared well for the next few years.

She presented to us again at the age of 11 with severe headache after having suffered a ground-level fall 5 days earlier. MRI demonstrated bilateral subdural hygromas presumably related to the rupture of the middle cranial fossa cyst (Fig. 2A and B). As a temporizing measure, bilateral burr holes were made for drainage of hygroma fluid, which was under high pressure. An external subdural drain was placed, but she could not be weaned from it over the next few days. She then underwent placement of a right subdural peritoneal shunt (SPS) with a programmable Strata valve (Medtronic) set at 0.5 . She initially did well; however, 3 weeks later she presented with a methicil-

ABBREVIATIONS OI = osteogenesis imperfecta; $S P S=$ subdural peritoneal shunt; VPS = ventriculoperitoneal shunt. 
Ho and Jane Jr.

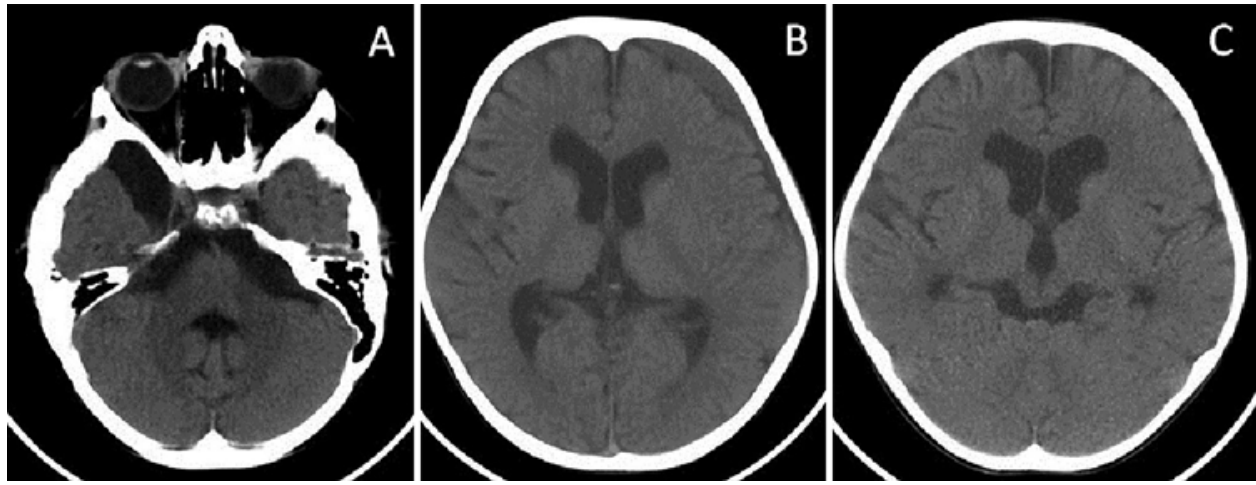

FIG. 1. Axial head CT images obtained at the initial presentation when the patient was 7 years old after a minor head injury, demonstrating an incidental right middle cranial fossa arachnoid cyst (A) and a subacute left subdural hematoma (B). By the 12-month follow-up (C), the subdural hematoma had resolved with conservative management. It was noted at the time that the patient had mild ventriculomegaly and sulcal prominence thought to be attributable to cerebral atrophy.
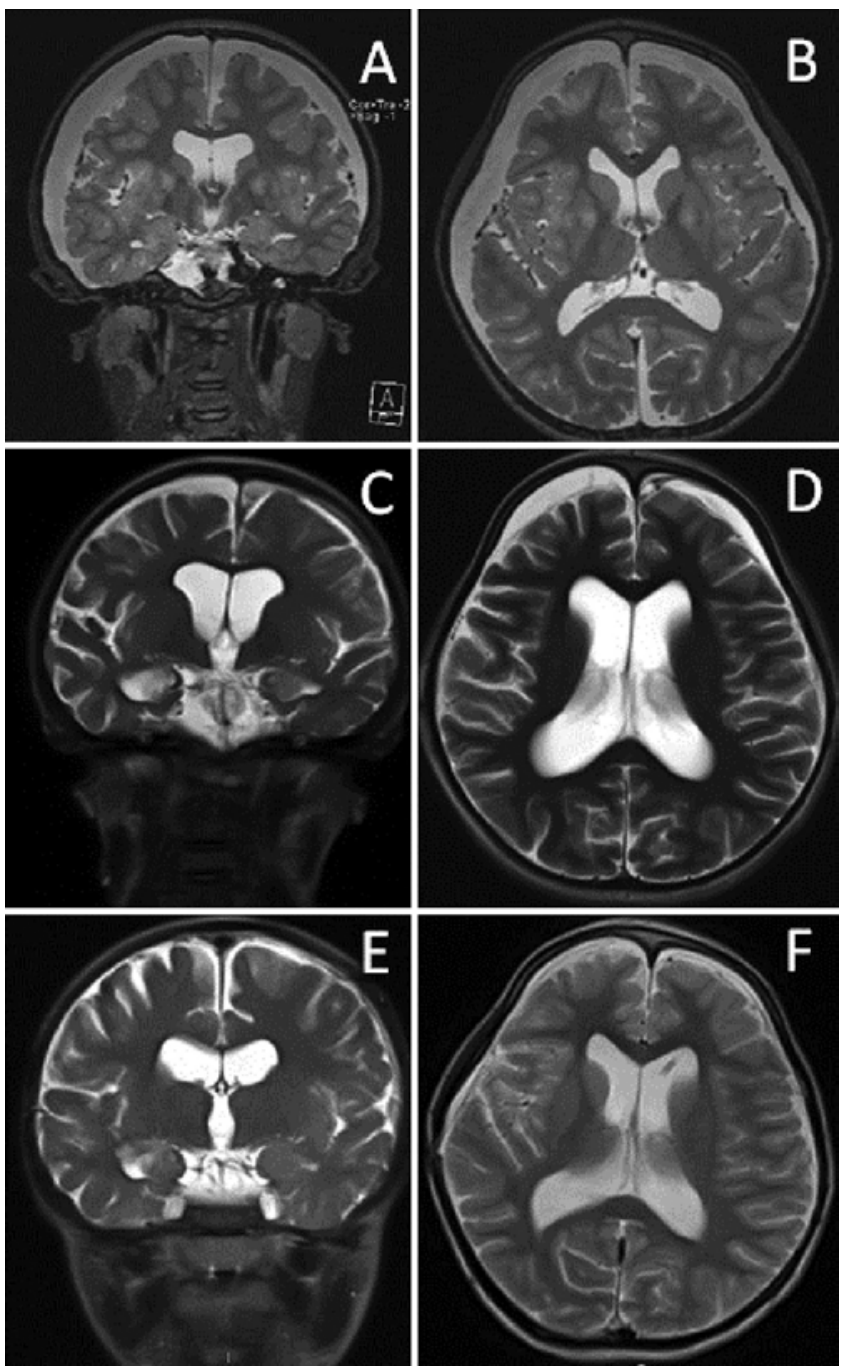

FIG. 2. Coronal and axial T2-weighted MR images (A and B) obtained when she was 11 years old after she had suffered a ground-level fall 5 days prior to her presentation with severe headache, demonstrating bilateral extraaxial fluid collection causing mass effect. Images ( $\mathbf{C}$ and D) obtained 7 weeks after right frontal SPS placement, demonstrating resolution of the subdural collection but increased ventricular size. Images (E and F) obtained 17 weeks after left frontal VPS placement, demonstrating decreased ventricular size. lin-sensitive Staphylococcus aureus (MSSA) shunt infection requiring explantation of the shunt and placement of an external subdural drain. After clearance of the CSF, she underwent reimplantation of the SPS with the Strata valve set at 0.5 . Unfortunately, she continued to be symptomatic with headache and emesis, requiring revision and replacement of the valve with a fixed ultra-low pressure valve. For 7 days, she was observed with an ICP monitor and was subsequently discharged with normal ICP measurements.

About 2 weeks later, she presented again with headache and emesis. MRI demonstrated a resolved subdural collection but increased ventriculomegaly (Fig. 2C and D). Given her clinical symptoms and worsened radiographic findings, we believed that she had developed "internal hydrocephalus" and recommended placement of a left frontal ventriculoperitoneal shunt (VPS) with a Strata valve set at 2.0. She did well after shunt placement and was followed up in clinic. At the 4-month follow-up, MRI demonstrated decreased ventricle size and resolution of the subdural hygroma (Fig. 2E and F). At the 2-year follow-up after VPS placement, she was noted to have progressive "band-like" collapse in the occipital area (Fig. 3). She was asymptomatic at that time; therefore, we recommended continued observation. However, over the following 6 months, she became increasingly symptomatic. She had progressive daily headaches that affected her sleep and increasing tenderness to touch in the affected areas. A CT venogram was obtained to confirm patency of the sagittal sinus (Fig. 4A). Three-dimensional reconstruction of head CT demonstrated collapse of her skull in the region of her lambdoid suture where she had significant Wormian bone formation (Fig. 4B and C). Given that her symptoms were significantly affecting her quality of life, we recommended a cranioplasty using a custom titanium mesh.

\section{Operation}

Surgery was performed with a bilateral parietal-occipital craniectomy encompassing the deformed area. Pairs of burr holes next to the sagittal sinus, superior and inferior to the deformity, were made. We then performed bilateral parietal-occipital craniectomies of the lateral margins of the Wormian bone. We left the Wormian bone over the sagittal sinus because of its adherence to the superior sag- 

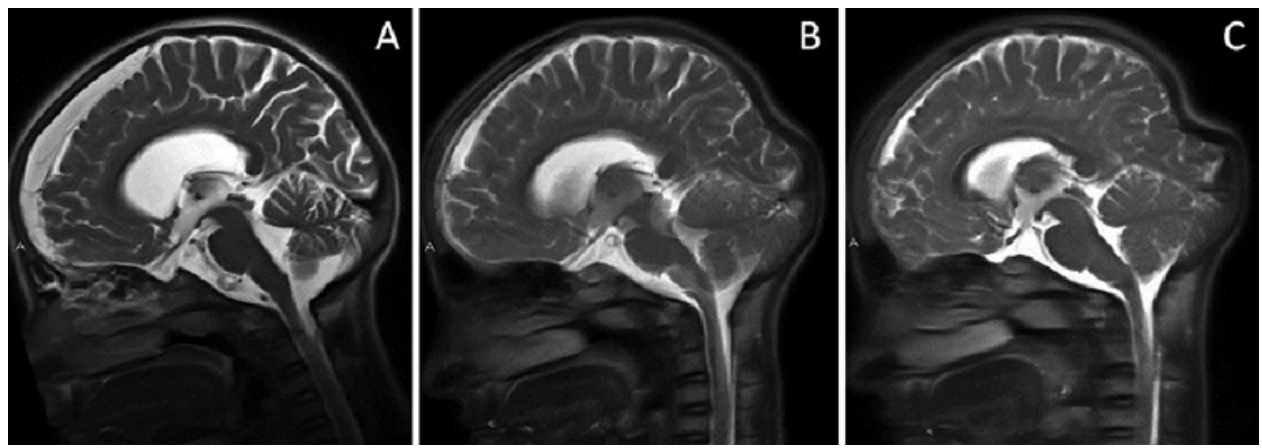

FIG. 3. Sagittal T2-weighted MR images obtained immediately (A), 10 months (B), and 21 months (C) after left frontal VPS placement.

ittal sinus in order to avoid any vascular injury. The custom titanium mesh was then placed to cover the defect.

\section{Postoperative Course}

Postoperative head CT demonstrated an excellent cosmetic outcome (Fig. 5). The patient had an uneventful postoperative course and was discharged on postoperative day 5. By the 3-month follow-up in clinic, her headaches had completely resolved and she was otherwise doing well clinically. At the 18-month follow-up, she continued to do well. The deformity was well corrected. MRI of the brain demonstrated stable ventricular size and continued resolved subdural collection.

\section{Discussion}

To our knowledge, this is the first case report documenting severe cranial deformity as a delayed complication of CSF diversion in a patient with OI. It is likely that the combination of over-shunting, from both an SPS and a VPS, and increased fragility of the skull from the Wormian bone associated with OI resulted in the formation of a progressive cranial deformity. Not only was the deformity significant from a cosmesis standpoint, but it was also causing severe nonpositional headache and tenderness on palpation. The surgery with titanium cranioplasty appeared to reverse her symptoms. The pathophysiological basis of her pain is rather intriguing. We believe that the headache was not the result of CSF hypotension given the headache's nonpositional nature and the fact that cranioplasty, which presumably did not address the underlying over-shunting, improved her symptoms. We can speculate that the pain was the result of the progressive cranial deformity generating tension on the pain-sensitive dura. The uniqueness of this situation precludes an informed discussion of the pain etiology based on the literature. A somewhat analogous situation could be seen in cases of fibrous dysplasia involving the skull base and/or temporal bone, in which the expansile fibrodysplastic lesions can similarly cause impingement of the dura. In fact, case series of fibrous dysplasia involving the skull showed a high incidence of headaches (approximately 60\%) as a presenting symptom. ${ }^{4,6}$ However, in those cases, lesions were generally chronic and not as rapidly progressive as in our case. The independent pathophysiology of fibrous dysplasia may also contribute to the generation of headache, making such a comparison largely speculative.

It is also important to point out that since our patient did not undergo ICP monitoring after VPS placement, we cannot definitively state that she had CSF hypotension from the two shunts. It was also impossible to distinguish which of the two shunts or whether it was the combination of the two that was ultimately responsible for causing the deformity. However, since the SPS was connected to a fixed ultra-low pressure valve while the VPS was connected to a Strata valve set at a relatively high setting of 2.0, one could speculate that the SPS was more likely the dominant contributing factor. It is also important to note that the cranioplasty had not altered the underlying CSF dynamics. If over-shunting were the culprit of deformity formation, the patient would be at continued risk of developing a deformity in other areas of the skull. If a deformity were to
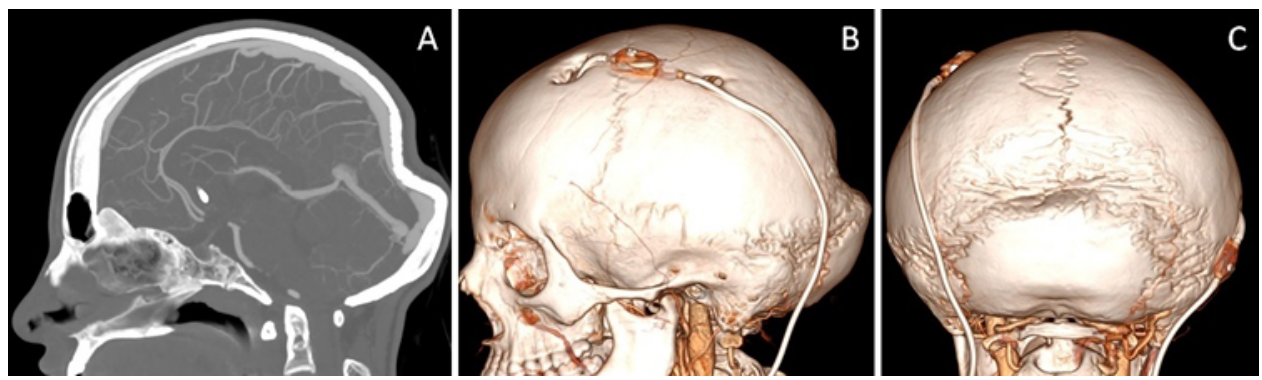

FIG. 4. Sagittal and 3D reconstruction of head CT venogram. Image (A) obtained 30 months after VPS placement, demonstrating patency of the sagittal sinus. Reconstruction images ( $B$ and $\mathbf{C}$ ) demonstrating the severe cranial deformity. Figure is available in color online only. 

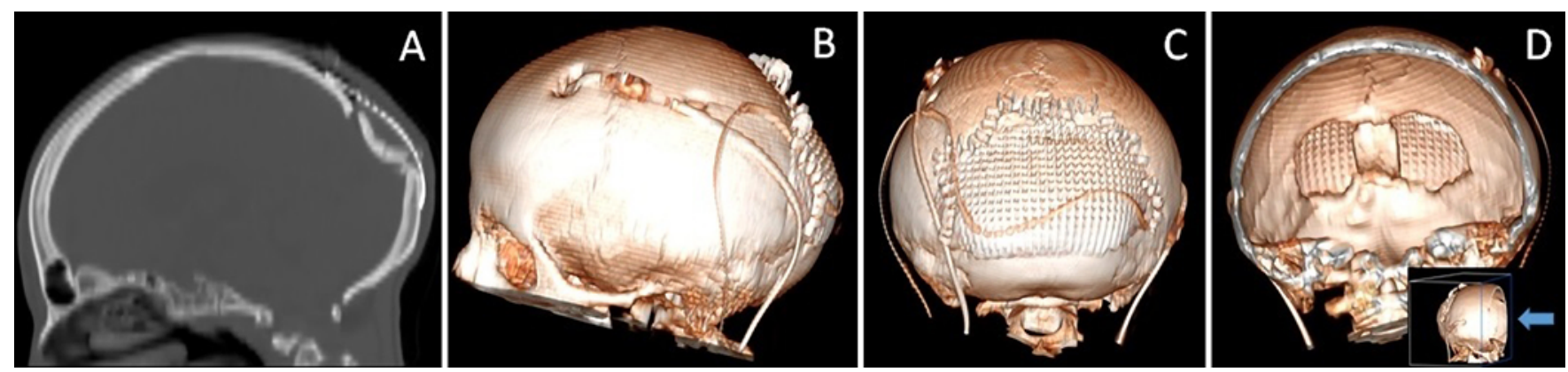

FIG. 5. Sagittal and 3D reconstruction of head CT. Images (A-C) obtained immediately after bilateral occipital craniectomy and cranioplasty with a custom titanium mesh, demonstrating correction of the cranial deformity. Anterior to posterior view (D) of the cranioplasty with cutoff of the anterior skull, demonstrating the residual midline Wormian bone. Three-dimensional orientation of the cutoff view (inset, D). Figure is available in color online only.

occur in the future, revision of the SPS and/or VPS would be indicated to decrease the amount of CSF shunting.

In conclusion, cranial deformity in an OI patient is a previously unrecognized complication following CSF diversion, which could be precipitated by over-shunting by a VPS or an SPS.

\section{References}

1. Charnas LR, Marini JC: Communicating hydrocephalus, basilar invagination, and other neurologic features in osteogenesis imperfecta. Neurology 43:2603-2608, 1993

2. Diaz LA, Lippe K: Acute extradural haematoma following trivial trauma in a case of osteogenesis imperfecta. Neurochirurgia (Stuttg) 28:180-181, 1985

3. Forlino A, Marini JC: Osteogenesis imperfecta. Lancet 387:1657-1671, 2016

4. Frisch CD, Carlson ML, Kahue CN, Pelosi S, Haynes DS, Lane JI, et al: Fibrous dysplasia of the temporal bone: a review of 66 cases. Laryngoscope 125:1438-1443, 2015

5. Groninger A, Schaper J, Messing-Juenger M, Mayatepek E, Rosenbaum T: Subdural hematoma as clinical presentation of osteogenesis imperfecta. Pediatr Neurol 32:140-142, 2005

6. Lustig LR, Holliday MJ, McCarthy EF, Nager GT: Fibrous dysplasia involving the skull base and temporal bone. Arch Otolaryngol Head Neck Surg 127:1239-1247, 2001

7. Menezes AH: Specific entities affecting the craniocervical region: Down's syndrome. Childs Nerv Syst 24:1165-1168, 2008

8. Mondal RK, Mann U, Sharma M: Osteogenesis imperfecta with bleeding diathesis. Indian J Pediatr 70:95-96, 2003
9. Morton ME: Excessive bleeding after surgery in osteogenesis imperfecta. Br J Oral Maxillofac Surg 25:507-511, 1987

10. Pozzati E, Poppi M, Gaist G: Acute bilateral extradural hematomas in a case of osteogenesis imperfecta congenita. Neurosurgery 13:66-68, 1983

11. Sasaki-Adams D, Kulkarni A, Rutka J, Dirks P, Taylor M, Drake JM: Neurosurgical implications of osteogenesis imperfecta in children. Report of 4 cases. J Neurosurg Pediatr 1:229-236, 2008

12. Shapiro JR, Sponsellor PD: Osteogenesis imperfecta: questions and answers. Curr Opin Pediatr 21:709-716, 2009

\section{Disclosures}

The authors report no conflict of interest concerning the materials or methods used in this study or the findings specified in this paper.

\section{Author Contributions}

Conception and design: Jane. Acquisition of data: Ho. Analysis and interpretation of data: Ho. Drafting the article: Ho. Critically revising the article: both authors. Reviewed submitted version of manuscript: both authors. Study supervision: Jane.

\section{Correspondence}

John A. Jane Jr.: University of Virginia Health System, Charlottesville, VA.johnjanejr@virginia.edu. 